\title{
Effect of a high fat diet on lipid absorption and fatty acid transport in a rat model of short bowel syndrome
}

Accepted: 10 December 2002/Published online: 30 April 2003

(C) Springer-Verlag 2003

\begin{abstract}
Long chain fatty acids (LCFAs) appear to be powerful stimulants for small bowel adaptation in patients with short bowel syndrome (SBS). However, the dietary lipid content may alter intestinal lipid transport. The aim of this study was to investigate the effects of a high fat diet (HFD) on in vivo lipid absorption and molecular and cellular mechanisms of LCFAs uptake by the remaining bowel. Male Sprague-Dawley rats (240-280) were randomly assigned to one of three groups: sham rats fed normal chow (sham-NC), SBS rats fed NC (SBS-NC) and SBS rats fed HFD (SBSHFD). SBS rats underwent a $75 \%$ small bowel resection. Rats were sacrificed on day 3 or 14 . Body weight, fat intake and fat clearance (total fecal fat) were measured twice a week. Fat absorbability was calculated as intake minus clearance and was expressed as percent of intake. Total RNA from the mucosa of duodenum, jejunum and ileum was extracted using TRIZOL Reagent. Northern blot analysis was performed to determine FAT/CD36 mRNA levels. Enterocyte LCFA transport was measured on day 14. LCFA uptake was determined by measuring cellular $[3 \mathrm{H}]$-oleate uptake over time (4-120 s). Mean ( \pm SE) FAT/CD36 mRNA levels and oleate uptake kinetic parameters were analyzed using ANOVA. Fat absorbability diminished after bowel resection, suggesting fat malabsorption. Remaining bowel in SBS-NC rats responded by an increase in FAT/ CD36 mRNA levels in the duodenum and ileum on day 3 , and the duodenum and jejunum on day 14 compared to sham-NC animals, and was accompanied by an increase in enterocyte LCFA transport in all segments. Exposure to a HFD for 14 days resulted in significantly
\end{abstract}

I. Sukhotnik $(\bowtie) \cdot$ A. S. Gork $\cdot$ M. Chen $\cdot$ R. A. Drongowski

A. G. Coran ' C. M. Harmon

C.S. Mott Children's Hospital and

University of Michigan Medical School,

Ann Arbor, MI, USA

E-mail: igor-dr@internet-zahav.net

Tel.: + 972-4-8250294

Fax: +972-4-8346083 increased fat absorbability after 3 days compared to SBS-NC rats. However, FAT/CD36 mRNA levels (vs. SBS-NC) decreased in all segments on day 3. On day 14, FAT/CD36 mRNA levels were decreased in the duodenum and ileum and were accompanied by reduced oleate uptake by isolated enterocytes in the ileum (vs. SBS-NC). In a rat model of SBS, early high fat diet increased lipid absorptive capacity of the intestinal remnant as seen by increased fat absorbability. The main mechanisms of this effect may be an acceleration of structural intestinal adaptation resulting in an increased number of enterocytes. However, at molecular and cellular levels HFD decreased mucosal FAT/CD36 mRNA levels and oleic acid uptake by isolated enterocytes.

Keywords Short bowel syndrome - Dietary lipid · Lipid absorption $\cdot$ Fatty acid uptake

\section{Introduction}

The ability of the bowel to digest and absorb enteral nutrients can be impaired in many diseases, including short bowel syndrome, with lipid absorption generally considered the most compromised. The short bowel syndrome (SBS) is a disorder in which either a loss of intestinal length or competence significantly compromises the ability to digest and absorb nutrients [1]. The key to survival after massive small bowel resection is the ability of the residual bowel to adapt. Intestinal adaptation begins within $48 \mathrm{~h}$ of resection and lasts for a least 18 months, and includes morphologic and functional changes in the residual bowel. Anatomical changes include lengthening of the villi, deepening of the crypts, increasing rates of enterocyte proliferation, increasing epithelial cell number/villus and, finally, an increase in the total number of enterocytes. Functional changes result in increasing nutrient uptake by isolated enterocytes. Several experiments have demonstrated that animals with resected intestine can often achieve virtually 
complete nutrient absorption by increasing their transport rates per centimeter of intestine [2]. Although lipid absorption in SBS is usually significantly compromised [3], little is known about the changes in the total absorptive capacity of the residual bowel for enteral lipids. Under normal conditions, long chain fatty acids (LCFA) can readily pass phospholipid membranes by passive diffusion [4]. However, recent kinetic studies have suggested that at least a portion of fatty acid cellular uptake may be carrier mediated $[5,6]$. Several proteins have been proposed as candidates for plasma membrane LCFA transporter proteins. Two of them are expressed in the small intestine: membrane fatty acid binding protein and fatty acid translocase (FAT), the rat homologue of human CD36. Recently we have demonstrated the upregulation of FAT and increased fatty acid transport in isolated enterocytes during intestinal adaptation in a rat with SBS [7]. The purpose of the present study was to evaluate the effect of dietary lipids on in vivo fat absorption in a rat following massive small-bowel resection and to investigate molecular and cellular mechanisms of this effect. Are the changes in the lipid absorption rate due to changes in the number of existing enterocytes or to the production of new cells with different transport activity? To resolve this problem, we investigated the effects of a high fat diet on structural intestinal adaptation in the first 2 weeks following massive small bowel resection in a rat. We also studied the effect of a high fat diet on FAT/CD36 expression in the remaining small bowel and on LCFA uptake by isolated enterocytes.

\section{Materials and methods}

Male Sprague-Dawley rats weighing $250-320 \mathrm{~g}$ were housed in individual, screen-bottomed cages. Constant environmental conditions (temperature $22^{\circ} \mathrm{C}$, humidity 50 to $55 \%$, and 12 -h light/ dark cycle) were maintained. Animals were given free access to standard rat chow (PMI Nutrition International) and water. After a 48-72 $\mathrm{h}$ acclimatization period, the rats were randomly assigned to one of three groups: (1) sham operation control rats fed normal chow (sham-NC), (2) rats with SBS fed normal chow (SBS-NC) and (3) rats with SBS fed a high fat diet (SBS-HFD).

The rats were anesthetized with intraperitoneal sodium pentobarbital (45 mg/kg) (The Butler Co., Columbus, Ohio) after an overnight fast. The abdomen was opened using a midline abdominal incision. For SBS rats, the small bowel was resected between a point $5 \mathrm{~cm}$ distal to the ligament of Trietz and $10 \mathrm{~cm}$ proximal to the ileo-cecal junction. The mesenteric blood supply was tied off with $5 / 0$ silk. An end-to-end single layer anastomosis was performed using $6 / 0$ silk. For sham animals, the small bowel was transected and anastomosed at a site $15 \mathrm{~cm}$ proximal to the ileo-cecal junction. For all operations, the abdominal cavity was closed in two layers with a running suture of $3 / 0$ Dexon. Antimicrobial cream with pramoxine $\mathrm{HCl}$ (Mycitracin Plus, Johnson and Johnson) was applied to the incision for topical pain relief.

Water was provided ad libitum for $24 \mathrm{~h}$ following the operation. Then the rats were given normal (10 kcal \% fat) or high fat (50 kcal \% fat) diets (Research Diets, Inc.; Table 1) and water ad libitum until they were killed. Following the operation, body weight and consumed food were weighed twice weekly. Food intake was calculated and expressed as grams per day. Feces from
Table 1 Composition of experimental diets

\begin{tabular}{lll}
\hline Ingredients $(\mathrm{g} \%)$ & Normal chow & High fat diet \\
\hline Casein, 80 Mesh & 200 & 200 \\
L-cystein & 3 & 3 \\
Corn starch & 315 & 22.5 \\
Maltodextrin 10 & 35 & 125 \\
Sucrose & 350 & 147.5 \\
Cellulose, BW200 & 50 & 50 \\
Soybean oil & 25 & 25 \\
Lard & 20 & 200 \\
Minerals* & 45 & 45 \\
Vitamins** & 12 & 12 \\
Total & $4.3 / 10$ & $27.1 / 49.9$ \\
Fat $\mathrm{g} \% / \mathrm{kcal} \%$ & 3.85 & 4.89 \\
Energy kcal/g & &
\end{tabular}

*Provided $(\mathrm{g} \%)$ : standard salt mix (S10001) 10.0, dicalcium phosphate 13.0, calcium carbonate 5.5, potassium citrate 16.5 **supplied $(\mathrm{mg} \%)$ : standard vitamin mix (V10001) 10.0, choline bitartrats 2.0

each animal were collected during the 14 days of the experimental period, weighed and frozen at $-18^{\circ} \mathrm{C}$ until analysis. Fecal lipid was extracted using ethanol and petroleum ether as described by Henry [8]. Energy and fat intake were calculated from the amount of ingested food. The difference between the amount of food consumed and the amount of the dry feces was used as an estimate of absorbability. The difference between the total milligrams of fat ingested and the total milligrams of fat remaining in the dry feces represented fat absorbability as previously described by other investigators [9].

Northern blot analysis

Animals were anesthetized with sodium phenobarbital intraperitoneally $(45 \mathrm{mg} / \mathrm{kg}$ ) and killed by open pneumothorax 3 or 14 days postoperatively. Intestinal mucosa was harvested, weighed and frozen in liquid nitrogen. RNA was isolated from mucosa using the TRIzol reagent (GIBCO BRL) as described by Chomczynski and Sacchi [10]. RNA was quantified by spectrophotometry at $\mathrm{A}_{260}$. Total RNA ( $30 \mu \mathrm{g} /$ lane) was separated on a $1 \%$ agarose gel containing formaldehyde $(18 \%)$, transferred to a nylon membrane using the Rapid Downward Transfer System (Schleicher and Schuell) and cross-linked to the membrane by exposure to ultraviolet waves for $20 \mathrm{~min}$ and by baking for $2 \mathrm{~h}$ at $80^{\circ} \mathrm{C}$. The membrane was pre-hybridized with $50 \%$ formamide, $5 \times \mathrm{SSPE}, 12 \%$ Denhardt's reagent, $0.1 \%$ SDS, $200 \mu \mathrm{g} / \mathrm{ml}$ salmon sperm overnight at $42^{\circ} \mathrm{C}$ and then was hybridized with 32P-labelled FAT cDNA probe (Rediprime labeling kit, Amersham Life Science) in 50\% formamide, $5 \times \mathrm{SSPE}, 2 \%$ Denhardt's reagent, $0.1 \%$ SDS, $200 \mu \mathrm{g} / \mathrm{ml}$ salmon sperm for $20 \mathrm{~h}$ at $42^{\circ} \mathrm{C}$. After two or three washes in $2 \times \mathrm{SSC}$ and $0.1-0.5 \%$ SDS at room temperature, the membrane was exposed to X-ray film (Kodak) for 24-72 $\mathrm{h}$. The 32P-labeled FAT cDNA probe was removed from the membrane (boiled $15 \mathrm{~min}$ in $0.1 \times \mathrm{SSC} / 0.5 \%$ SDS), and the membrane was reprobed with $32 \mathrm{P}$-labeled $18 \mathrm{~s}$ cDNA probe. Autoradiographs were analyzed by densometry using a Hewlett Packard ScanJet 4c/T in conjuction with NIH Image software (version 1.60).

\section{Isolation of intestinal cells}

The method of Gore was used to isolate the cells from the remaining bowel of experimental rats [11]. At sacrifice small intestine from the pylorus to the ileo-cecal valve was removed and divided into three segments: duodenum, jejunum to anastomosis and terminal ileum. The luminal contents were rinsed in Hank's 
medium at $37^{\circ} \mathrm{C}$. The isolated intestinal segments were filled with medium A $(3 \mathrm{mmol} / \mathrm{l}$ phosphate buffer/136 mmol/1 NaCl/ $0.6 \mathrm{mmol} / 1 \quad \mathrm{CaCl}_{2} / 5.2 \mathrm{mmol} / 1 \mathrm{KCl} / 0.8 \mathrm{mmol} / 1 \quad \mathrm{MgSO}_{4} / 5 \mathrm{mmol} / 1$ glucose, $\mathrm{pH}$ 7.3) and submersed in Hank's medium for $15 \mathrm{~min}$ at $37^{\circ} \mathrm{C}$ with oxygenation. The medium was then emptied, filled with solution B $\left(140 \mathrm{mmol} / 1 \quad \mathrm{NaCl} / 16 \mathrm{mmol} / 1 \quad \mathrm{Na}_{2} \mathrm{HPO} 4 / 1.5 \mathrm{mmol} / \mathrm{l}\right.$ EDTA $/ 0.5 \mathrm{mmol} / 1$ dithiothreitol, $\mathrm{pH} 7.3$ ) and incubated for $3 \mathrm{~min}$ at $37^{\circ} \mathrm{C}$ under oxygenation, after which it was gently palpated for 2 min. The treatment with solution B was repeated twice. After isolation, the mucosal cells were filtered through $0.25 \mathrm{~mm}$ mesh gauze, centrifuged at $70 \mathrm{~g}$ for $2 \mathrm{~min}$ and washed twice with Hank's medium. Thereafter, the cells were diluted to $2 \times 10^{6}$ cells $/ \mathrm{ml}$ in Hank's medium.

Cellular uptake of $\left[{ }^{3} \mathrm{H}\right]$ oleic acid

Uptake of $\left[{ }^{3} \mathrm{H}\right]$-oleic acid (Sigma) was measured by rapid vacuum filtration assay. One hundred twenty-five $\mu \mathrm{l}$ of cellular stock suspension was mixed with $125 \mu \mathrm{l}$ of $\left[{ }^{3} \mathrm{H}\right]$-oleic acid/taurocholate solution in polypropylene tubes. At various time points $(4,8,15$, $30,60,90$ and $120 \mathrm{~s}), 5 \mathrm{ml}$ of ice-cold $0.5 \%$ albumin in incubation medium (BSA/KRH) was pipetted into $250 \mu \mathrm{l}$ sample aliquots to stop cellular influx and efflux and to remove surface-bound fatty acids. The stop solution containing cells was quickly pipetted onto the center of a Whatman GF/C microfiber filter (24 mm), and filtered under $50 \mathrm{~mm} \mathrm{Hg}$ vacuum pressure using a filtration apparatus (model 8010, Millipore Stirred Cells, Amicon Bioseparations). The cells were washed twice with $1 \mathrm{ml}$ of $0.5 \%$ albumin in incubation medium. The filters were placed in scintillation vials, $10 \mathrm{ml}$ of scintillation fluid (EcoLite, ICN; Costa Messa, Calif.) was added and the radioactivity was determined in a liquid scintillation counter (Beckman LS 1801, Irwin, Calif.). Nonspecific association of activity to filters and cells was determined in each experiment by adding the cold stop solution before the addition of corresponding aliquots of cells and $\left[{ }^{3} \mathrm{H}\right]$-oleic acid working solutions. The value of each sample was the net radioactivity after subtraction of this blank.

Statistical analysis

Results of FAT/CD36 mRNA levels are expressed as mean percent $( \pm$ SEM) of the control ribosomal $18 \mathrm{~S}$ mRNA levels of each sample. Initial rates of oleate uptake were determined from the best-fit curve for time course data utilizing non-linear regression analysis. Statistical significance for mean value \pm SEM of FAT/ CD36 mRNA levels and kinetic parameters ( $K_{\mathrm{m}}$ and $\left.V_{\max }\right)$ was determined using the Student's t-test. $P$ values less than 0.05 were considered significant.

\section{Results}

Body weight

A significant decrease in body weight occurred on day 3 after bowel resection in rats fed both normal chow and a high fat diet compared to their control counterparts. After the third postoperative day, SBS rats fed different diets gained weight similarly. There was a significant difference in the final weight between resected and sham$\mathrm{NC}$ animals, but no significant difference between SBS rats fed different diets (Table 2). The animals did not develop significant diarrhea following bowel resection.

\section{Food and fat balance}

Food balance in different experimental groups is presented in Table 2. Calculated food absorbability did not change significantly among all experimental groups on day 3. On day 14, food intake was decreased in SBS-NC rats (vs. sham-NC animals). However, food clearance (dry fecal mass) remained unchanged. Therefore, calculated food absorbability decreased in SBS-NC, suggesting generalized malabsorption. Early exposure to HFD was associated with a decrease in food intake and non-proportionally greater decrease in food clearance. Consequently, calculated food absorbability increased significantly in SBS-HFD rats (vs. SBS-NC), suggesting that HFD improves generalized malabsorption in this model.

In accordance with food intake, a small but significant decrease in fat intake was seen in SBS-NC rats compared to the sham-NC group on day 3 (Table 2). However, fat clearance remained unchanged. Consequently, calculated fat absorbability decreased in SBS-NC rats (vs. sham-NC), suggesting fat malabsorption. On day 14, SBS-NC rats demonstrated fat intake similar to sham-NC, but greater fat clearance (steatorrhea). Consequently, fat absorbability was lower again in SBS-NC rats compared to their control counterparts.

Table 2 Food, fat and energy balance. Average final body weight, food and fat intake, clearance and absorbability in normal and operated rats (sham and SBS rats) fed normal chow or high fat diet for 2 weeks. Values are mean \pm SEM. SBS short bowel syndrome, $N C$ normal chow, $H F D$ high fat diet

\begin{tabular}{|c|c|c|c|c|c|c|}
\hline \multirow[t]{2}{*}{ Parameters } & \multicolumn{3}{|l|}{ Day3 } & \multicolumn{3}{|l|}{ Day14 } \\
\hline & Sham-NC & SBS-NC & SBS-HFD & Sham-NC & SBS-NC & SBS-HFD \\
\hline Body weight (\% preoperative) & $99.7 \pm 1.5$ & $91.3 \pm 2.3^{\mathrm{a}}$ & $90.9 \pm 1.8^{\mathrm{a}}$ & $130.6 \pm 3.7$ & $118.1 \pm 3.4^{\mathrm{a}}$ & $113.9 \pm 2.7^{\mathrm{a}}$ \\
\hline Energy intake $(\mathrm{kcal} / \mathrm{d})$ & $51.3 \pm 8.4$ & $45 \pm 7.8$ & $31.1 \pm 4.7^{\mathrm{a}}$ & $97.4 \pm 6.7$ & $82 \pm 5.3$ & $76 \pm 8.4^{\mathrm{a}}$ \\
\hline Food intake $(\mathrm{g} / \mathrm{d})$ & $13.3 \pm 2.2$ & $11.7 \pm 2.0$ & $6.4 \pm 1.0^{\mathrm{a}, \mathrm{b}}$ & $25.3 \pm 0.7$ & $21.9 \pm 1.4^{\mathrm{a}}$ & $15.6 \pm 1.7^{\mathrm{a}, \mathrm{b}}$ \\
\hline Fecal mass $(\mathrm{g} / \mathrm{d})$ & $3.3 \pm 0.5$ & $3.1 \pm 0.5$ & $1.4 \pm 0.2$ & $6.2 \pm 0.5$ & $6.0 \pm 0.5$ & $2.2 \pm 0.2^{\mathrm{a}, \mathrm{b}}$ \\
\hline Food absorbability (\%) & $74.2 \pm 1.8$ & $72 \pm 1.8$ & $73.3 \pm 4.7$ & $75.6 \pm 0.6$ & $72.6 \pm 1.6^{\mathrm{a}}$ & $84.6 \pm 2.5^{\mathrm{a}, \mathrm{b}}$ \\
\hline Fat intake $(\mathrm{mg} / \mathrm{d})$ & $641 \pm 72$ & $459 \pm 63^{\mathrm{a}}$ & $1825 \pm 261^{\mathrm{a}, \mathrm{b}}$ & $1087 \pm 74$ & $1001 \pm 92$ & $4214 \pm 463^{\mathrm{a}, \mathrm{b}}$ \\
\hline Fat clearance $(\mathrm{mg} / \mathrm{d})$ & $78 \pm 10$ & $87 \pm 18$ & $148 \pm 42^{\mathrm{a}, \mathrm{b}}$ & $131 \pm 9$ & $198 \pm 21^{\mathrm{a}}$ & $263 \pm 59^{\mathrm{a}}$ \\
\hline Fat absorbability $(\%)$ & $88 \pm 0.8$ & $80.2 \pm 3.2^{\mathrm{a}}$ & $92.2 \pm 1.8^{\mathrm{b}}$ & $87.9 \pm 0.6$ & $79.4 \pm 2.5^{\mathrm{a}}$ & $92.3 \pm 2.5^{\mathrm{a}, \mathrm{b}}$ \\
\hline
\end{tabular}

${ }^{a} P<0.05$ SBS vs. sham-NC

b $P<0.05$ SBS-HFD vs. SBS-NC 


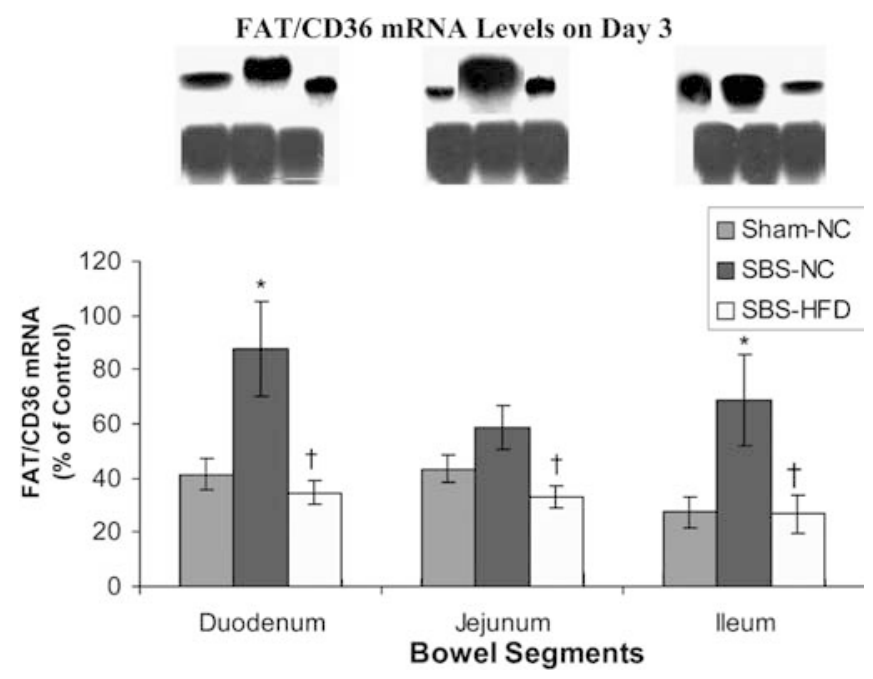

Fig. 1 Effects of a high fat diet and bowel resection on FAT/CD36 mRNA expression ( $\%$ of $18 \mathrm{~S}$ ) in duodenum, jejunum and ileum on day 3 of dietary treatment. Values are mean \pm SEM. $S B S$ short bowel syndrome, $N C$ normal chow, HFD high fat diet. $* P<0.05$ SBS vs. sham-NC, $\dagger P<0.05$ SBS-HFD vs. SBS-NC

Exposure to HFD resulted in a significant increase in fat intake and less significant increase in fat clearance on days 3 and 14 (vs. SBS-NC and sham-NC animals). Therefore, calculated fat absorbability was significantly greater in SBS-HFD rats compared with SBS-NC and sham-NC animals, suggesting that HFD improved invivo lipid absorption.

\section{FAT/CD 36 expression}

A significant twofold increase in the levels of FAT/CD36 mRNA was observed in the duodenum $(87.6 \pm 17.6$ vs. $41.3 \pm 5.7 \%, P<0.05)$ and ileum $(68.7 \pm 17.1$ vs. $27.4 \pm$ $5.6 \%, P<0.05)$ on day 3 following bowel resection in rats fed normal chow compared to sham-NC animals (Fig. 1). Early exposure to a high fat diet resulted in a marked decrease in FAT mRNA levels (vs. SBS-NC rats) in the duodenum $(34.6 \pm 4.2$ vs. $87.6 \pm 17.6 \%$, $P<0.05)$, jejunum $(33.0 \pm 4.0$ vs. $58.7 \pm 8.1 \%, P<0.05)$ and ileum $(26.8 \pm 7.1$ vs. $68.7 \pm 17.1 \%, \quad P<0.05)$, reaching values not different from sham-NC rats.

Northern blot analysis on day 14 showed a significant increase in FAT/CD36 mRNA levels in the duodenum $(58.6 \pm 8.7$ vs. $31.1 \pm 6.2 \%, P<0.05)$ and jejunum $(66.1 \pm 8.7$ vs. $43.4 \pm 8.4 \%, P<0.05)$ in SBS-NC rats compared to sham-NC animals (Fig. 2). Exposure to a high fat diet led to a significant decrease in FAT/CD36 levels in the duodenum $(25.6 \pm 4.5$ vs. $58.6 \pm 8.7 \%$, $P<0.05)$ and ileum $(32.7 \pm 7.4$ vs. $80.5 \pm 27.6 \%$, $P<0.05)$, but did not affect the levels in the jejunum.

Uptake kinetics of $\left[{ }^{3} \mathrm{H}\right]$-oleic acid

by isolated mucosal cells

As shown in Fig. 3 and Table 3, bowel resection in rats fed normal chow resulted in a significant increase

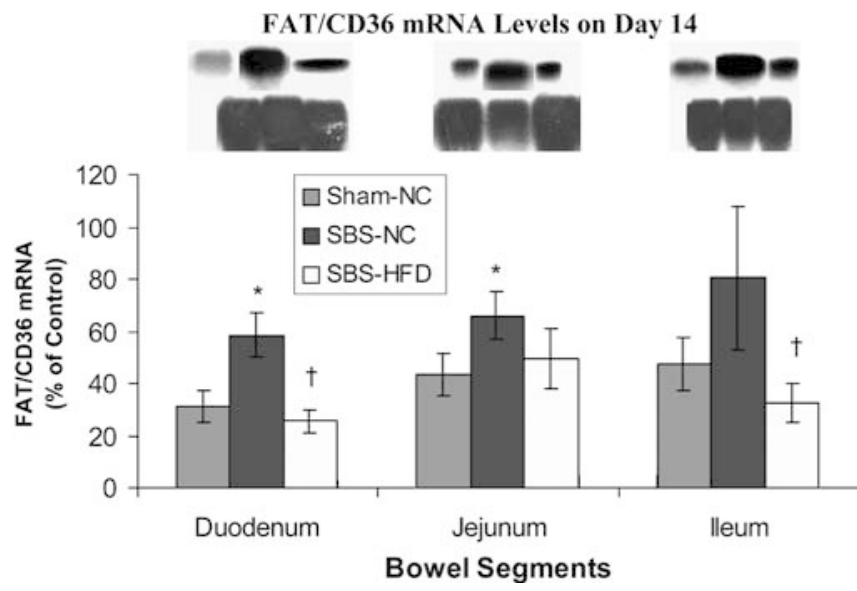

Fig. 2 Effects of a high fat diet and bowel resection on FAT/CD36 mRNA expression (\% of $18 \mathrm{~S}$ ) in duodenum, jejunum and ileum on day 14 of dietary treatment. Values are mean \pm SEM. SBS short bowel syndrome, $N C$ normal chow, $H F D$ high fat diet. * $P<0.05$ SBS vs. sham-NC, $\dagger P<0.05$ SBS-HFD vs. SBS-NC

(vs. sham-NC) in enterocyte $\left[{ }^{3} \mathrm{H}\right]$-oleate uptake rates as a function of LCFA concentration in the duodenum (Vmax $213 \pm 13$ vs. $68 \pm 8 \mathrm{nmol} / 10^{6}$ cells $/ \mathrm{min}, P<0.05$ ), jejunum (Vmax $357 \pm 19$ vs. $125 \pm 11 \mathrm{nmol} / 10^{6}$ cells $/ \mathrm{min}$, $P<0.05)$ and ileum (Vmax $380 \pm 23$ vs. $100 \pm 9 \mathrm{nmol} /$ $10^{6}$ cells $/ \mathrm{min}, P<0.05$ ) on day 14 following the operation. The transport $\mathrm{Km}$, increased in the duodenum $(0.5 \pm 0.02$ vs. $0.4 \pm 0.01 \mu \mathrm{mol} / 1, \quad P<0.05)$ and ileum $(0.2 \pm 0.01$ vs. $0.1 \pm 0.02 \mu \mathrm{mol} / 1, \quad P<0.05)$, suggesting decreased affinity of the ligand for its receptor. Early exposure to a high fat diet decreased significantly (compared to SBS-NC rats) oleate uptake by isolated enterocytes in the ileum (Vmax $222 \pm 15$ vs. $380 \pm 23 \mathrm{nmol} / 10^{6}$ cells $\left./ \mathrm{min}, \quad P<0.05\right)$ on day 14 following the operation. Km was significantly lower in SBS-HFD rats (vs. SBS-NC) in the duodenum $(0.1 \pm 0.02$ vs. $0.5 \pm 0.02 \mu \mathrm{mol} / 1, \quad P<0.05)$ and ileum $(0.16 \pm 0.01$ vs. $0.2 \pm 0.01 \mu \mathrm{mol} / 1, \quad P<0.05)$, suggesting increased affinity of the ligand for its receptor.

\section{Discussion}

Because of the general importance of LCFA in the diet it is to be expected that enterocytes should be able to regulate FA uptake in order to adapt to changes in energy demands [12]. Given their multiple roles in cell function, it is important to understand the mechanisms that mediate cellular uptake of LCFA. Because of their lipophilic nature, LCFAs are thought to diffuse freely through the plasma membrane. A recent review by Hamilton [4] presented the concept that passive diffusion through the lipid bilayer is the central mechanism of transport of LCFA into and out of cells; this mechanism guarantees that the necessary LCFAs are supplied to the cell and the excess LCFAs are removed.

However, recent studies have suggested that fatty acid absorption is, at least in part, a carrier-mediated 

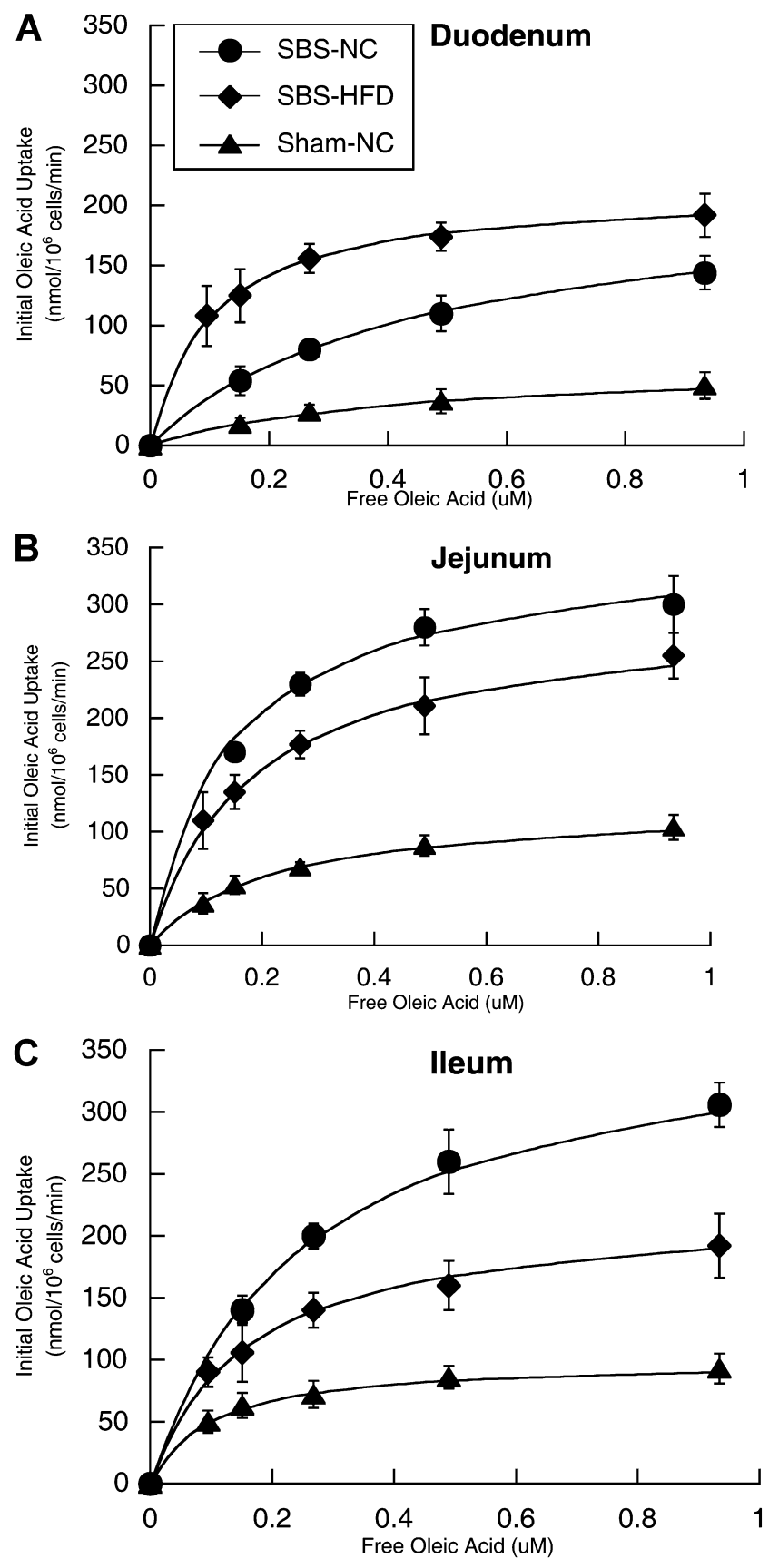

Fig. 3 Relationship between oleic acid monomers concentration in the incubation medium and initial uptake rate by isolated enterocytes in duodenum, jejunum and ileum in rats with short bowel syndrome fed normal chow (SBS-NC) or high fat diet (SBSHFD) vs. sham-NC animals. Values are mean \pm SE. $S B S$ short bowel syndrome, $N C$ normal chow, $H F D$ high fat diet. ${ }^{*} P<0.05$ SBS vs. sham-NC, $\dagger P<0.05$ SBS-HFD vs. SBS-NC

process $[5,12]$. Several proteins have been proposed as candidates for plasma membrane LCFA transporter proteins. At least two of them are expressed in the small intestine: plasma membrane fatty acid binding protein (FABPpm) and fatty acid translocase (FAT).

FAT is an $88 \mathrm{kDa}$ integral membrane protein and was identified at first in rat adipocytes by specific labeling with reactive sulfo-N-succinimidyl derivates of fatty acid under conditions where FA uptake was inhibited by about 75\% [13]. CD36 distribution favors tissues with a high metabolic capacity for LCFA (adipose, muscle, heart and intestine), while it is absent from tissues like brain that do not utilize exogenous LCFAs. Regulation of FAT/CD36 expression in the bowel is consistent with a role in LCFA transport. In normal circumstances, the intestinal absorption of fatty acids is responsive to changes in dietary fat content and to the presence of an intact intestinal tract.

Short bowel syndrome is a condition characterized by a loss of intestinal mass and function, resulting in a diminished ability to digest and absorb a regular diet. A combination of significant reduction in absorptive area and severely compromised enterohepatic circulation results in increasing steatorrhea and fat malabsorption. Despite compromised lipid absorption and associated problems with steatorrhea, the patients with SBS do not typically have clinical signs of fatty acid deficiency [1]. It is expected that intestinal cells should be able to regulate LCFA uptake in order to adapt to changes in energy demands. We recently have demonstrated that FAT/ CD36 mRNA levels are differentially increased along the gut axis during intestinal adaptation in a rat model of SBS [7]. In the present study, we investigated the early effects of massive small bowel resection and high fat diet on intestinal lipid absorption using a rat model of SBS.

In our experimental model, massive bowel resection in rats fed normal chow produced a significant decrease in food absorbability, suggesting food malabsorption, and a decrease in fat absorbability, suggesting fat malabsorption compared to control animals. Decreased body weight gain accompanied the changes in food and fat absorption rates signifying in vivo metabolic alterations secondary to SBS. In evaluating molecular and cellular mechanisms of LCFAs uptake following small bowel resection, we have shown that FAT mRNA levels in remaining bowel segments significantly increased followed bowel resection and was accompanied by an increased LCFA uptake by isolated small bowel enterocytes compared to sham animals. These results are consistent with our previous results and suggest that as a putative LCFA protein, FAT/ CD36 may play a specific role in facilitating increased LCFA absorption during intestinal adaptation [7].

Early exposure of SBS animals to a high fat diet led to a significant increase in fat absorbability. These results suggest that with exposure to increased dietary fat, the remaining bowel, after massive resection, responds in an effort to increase intestinal lipid absorptive capacity. Two possible mechanisms could explain increased fat absorbability: (1) an increase in intestinal epithelial cell mass, or (2) enhancement of LCFA uptake by enterocytes. It has been reported previously that early exposure to a high fat diet led to strong stimulation of structural adaptation following bowel resection in a rat $[14,15]$. These data suggested that small bowel enlargement stimulated by HFD is predominantly due to mucosal hyperplasia. In contrast, our experiment 
